\title{
Unequal Clustering Multipath Routing Algorithm for Wireless Sensor Network based on Clustering and Ant Colony
}

\author{
Yong-xian Jin \\ College of Mathematics and Computer Science, Zhejiang Normal University, Jinhua Zhejiang 321004, China
}

\begin{abstract}
To improve the energy efficiency of the wireless sensor network (WSN), and extend the network life. This paper proposes an improved unequal clustering multipath routing algorithm (UCMRA). The algorithm improves the formula of cluster head selection probability and competition radius, and considers the energy factor, node density, optimal number of cluster heads, etc. Experimental results show that, compared with the traditional algorithm, UCMRA has more stable cluster head distribution, less energy consumption and longer network lifetime.
\end{abstract}

Keywords: Wireless sensor network; Ant colony algorithm; Energy efficiency; multipath routing.

\section{INTRODUCTION}

Wireless sensor network is a convenient new selforganizing network, which has been widely used in military, industrial, medical and other fields. It is a kind of network composed of numerous cheap micro sensors. Due to the limited energy of sensor nodes and the interference of external environment, the network life cannot be guaranteed, and the reliability of data transmission is greatly reduced. Therefore, it is very important to design an energy efficient routing algorithm.

Wireless sensor routing mechanism can be classified according to the routing characteristics, generally can be divided into planar and hierarchical routing or single path and multi-path routing. A large number of research results show that hierarchical routing and multipath routing are more helpful to improve the energy efficiency of the network and balance the network load. In the hierarchical routing, the clustering algorithm divides the network nodes according to the hierarchy, and several adjacent nodes can form a cluster. Then each cluster needs to select a cluster head node $\mathrm{CH}$ (cluster head). The cluster head will be responsible for the communication of the nodes in the cluster, and provide appropriate routing information for the communication between clusters. The formation of clusters can greatly reduce the amount of data transmission and save node energy. In multipath routing, ant colony algorithm has gradually become a research hotspot. It is a distributed algorithm, which was first used to solve TSP (traveling salesman problem) [1]. In the process of foraging, ants send out a pheromone in order to leave food position signals to their peers. The more pheromones left in a path, the more ants passing through the path, the more likely they are to find food. Ant colony algorithm is to use this principle to establish the optimal path of data transmission.

At present, there are routing algorithms that combine non-uniform clustering with ant colony algorithm: The AC-EBUC (energy balanced uneven clustering algorithm based on ant colony for wireless sensor network) algorithm proposed in reference [3] is an algorithm that promotes non-uniform clustering and ant colony algorithm. In the clustering stage, time delay, bandwidth and other factors are considered on the basis of EEUC [2] algorithm, which improves the QoS of the network. However, after each round of data transmission, it needs to be clustered again, this increases the network overhead. The improved WSN non-uniform clustering routing algorithm proposed in reference [4] introduces the idea of node "degree" when selecting the cluster head. In the transmission process, the routing is established based on the minimum spanning tree, and the hybrid routing is used for communication. The algorithm is relatively stable and energy-efficient. The IAC-UC (ant colony based uneven clustering routing algorithm) algorithm proposed in reference [5] fully considers the link quality and energy related factors in the inter cluster path establishment stage, which makes the calculation of pheromone concentration more reasonable and effectively delays the network lifetime.

Considering the advantages and disadvantages of the above routing algorithms, an improved routing algorithm UCMRA is designed, which combines non-uniform clustering algorithm and ant colony algorithm. When selecting cluster heads, the algorithm takes full account of 
energy, node density, the optimal number of cluster heads and other factors, so as to reduce energy consumption and prolong the network cycle.

\section{UCMRA ALGORITHM}

\section{A. Clustering stage}

\section{(1) Generation of candidate cluster heads}

UCMRA algorithm uses the value of the generation range from 0 to 1 to compare with the threshold to generate candidate cluster heads. The algorithm applies the following threshold formula (1).

$T^{\prime}(n)=\left\{\begin{array}{lr}\frac{p}{1-p \times(r \bmod 1 / p)} \times\left(q_{1} \frac{E_{\text {res }}}{E_{\text {avg }}}+q_{2} \frac{N_{n}}{N}\right), & n \in G \\ 0, & n \notin G\end{array}\right.$

Where $p$ is the probability that the node is selected as the cluster head; $r$ represents the current round value; $G$ is the set of unselected nodes in the last round $1 / p ; q_{1}$ and $q_{2}$ are weight factors, and satisfy $q_{1}+q_{2}=1 ; E_{\text {res }}$ is the residual energy of the node; $E_{\text {avg }}$ is the average residual energy of nodes; $N_{n}$ is the number of surviving neighbor nodes; $N$ is the total number of surviving nodes. Due to the random deployment of wireless sensor nodes, the improved threshold formula takes into account the node density, that is, the ratio of the number of surviving neighbors to the total number of nodes within a certain radius of competition.

The candidate cluster head generation strategy is shown in equation (2), which is the random value generated by node $\mathrm{n}$, and the value range is $(0,1)$. As can be seen from equations (1) and (2), when the sum is larger, the threshold is larger, and the probability of selecting candidate node is larger, otherwise, the probability is smaller. If the random value of the node is greater than the threshold, the campaign will not continue.

$\left\{\begin{array}{l}u_{n} \leq T^{\prime}(n) ; \text { node } n \text { is selected as candidate node } \\ u_{n}>T^{\prime}(n) ; \text { node } n \text { abandons election }\end{array}\right.$

\section{(2) Calculate competition radius}

The EEUC algorithm only considers the distance length when calculating the competition radius of candidate cluster heads, We improve the formula as follows:

$R_{c}^{\prime}=\left[c_{1}\left(1-\frac{d_{\text {max }}-d_{n s}}{d_{\text {max }}-d_{\text {min }}} \times \frac{E-E_{\text {res }}}{E-E_{\text {min }}}\right)+c_{2}\left(1-\frac{N_{n}}{N}\right)\right] R_{\text {max }}$

Where, $c_{1}$ and $c_{2}$ are weight factors respectively, $c_{1}$ $+c_{2}=1 ; R \max$ it is the maximum competition radius set in advance. $d_{\max }$ and $d_{\min }$ are the maximum and minimum values of the sink nodes separated from each other; $d_{n s}$ represents the distance from the current node to the sink node; $E$ is the initial energy of the node; $E$ min is the bottleneck value of network energy.

The improved competition radius formula shows that the shorter $d_{n s}$, the more energy consumption and the higher the node density, then $R_{c}^{\prime}$ and the cluster size is he smaller. Compared with the traditional formula, the energy and the number of adjacent nodes is considered to further effectively alleviate the serious local energy consumption, balance the network load, avoid the premature paralysis of the local network, and improve the overall performance of the network.

\section{(3) Final cluster head election mode}

After the candidate cluster heads are generated, they form a set together, from which the final cluster head node needs to be determined. The number of cluster heads affects the energy consumption of the whole network. The optimal number of cluster heads is obtained according to formula (4) [6].

$$
k_{o p t}=\frac{\sqrt{N}}{\sqrt{2 \pi}} \sqrt{\frac{\xi_{f s}}{\xi_{a m p}}} \frac{M}{d^{2}{ }_{t o B S}}
$$

The candidate cluster head is the final cluster head with the largest ratio according to formula (5). Then, the cluster head sends the election message to the neighboring nodes within the range of their competition radius. The other nodes apply for the cluster themselves and list the cluster heads in the list of neighboring nodes (as shown in Table 1). The cluster head also includes the effective members in their own neighbor table to establish the neighbor relationship.

$$
\max \frac{E_{r e s}}{d_{n s}}
$$

Table 1 list of adjacent nodes

\begin{tabular}{cl}
\hline Identification & Describe \\
\hline ID & Node number \\
State & Node status \\
$E_{r e s}$ & Node residual energy \\
$R^{\prime}{ }_{c}$ & Node competition radius \\
$d$ & Distance from the node \\
\hline
\end{tabular}

\section{B. Multi-path establishment}

The multi hop mechanism is used in cluster head communication. After the cluster head is determined, the cluster head will send identity information and related information of adjacent nodes to the Sink node. The Sink 
node establishes the multi hop forwarding path through the optimized ant colony algorithm. If there is no initial information of routing between cluster head and Sink node, each cluster head will release the forward ant to establish routing.

This algorithm improves the traditional heuristic formula, as shown in equation (6). Among them, $E_{\text {res }}$ is the node residual energy; $E_{\text {cost }}$ is energy consumption of sending data to node $\mathrm{m}$ for node $\mathrm{n} ; d_{n m}$ and $d_{m s}$ are the distances from $\mathrm{n}$ to $\mathrm{m}$ and $\mathrm{m}$ to Sink, respectively; $\alpha$ and $\beta$ are weight factors respectively, satisfying $\alpha+\beta=1$. The traditional heuristic function only includes the distance factor of the next hop object, and the improved formula also adds energy and other factors. In this way, when selecting the next hop object, the forward ant has a higher probability of selecting the node with high energy, low energy consumption and closer to sink node, and its working life is longer.

$$
\eta_{n m}(t)=\frac{E_{\text {res }} / E_{\text {cost }}}{\alpha d_{n m}+\beta d_{m s}}
$$

Every time the forward ant arrives at the destination cluster head, it will immediately update the routing table of the cluster head, including the total emission energy consumption, residual energy and other data, and then convert to the backward ant return, it needs to complete the task of updating the pheromone concentration on the path. The pheromone concentration updating formula is improved as equation (7), where $E_{\min }$ is the minimum value of the residual energy of the node in the backward ant path, that is, the energy bottleneck value; $E_{\text {avg }}$ is the average energy consumed in the path; Hop is the total number of hops; is the time delay from Sink to this node. It can be seen that the pheromone concentration will change with the change of energy, hop number and delay value. The node with small energy bottleneck value, more hops and longer time has less pheromone concentration, so it is less likely to become the best routing member, and vice versa.

$$
\Delta \tau_{n m}^{k}(t)=\frac{E_{\text {min }}}{E_{\text {avg }} \times H o p \times T_{\text {delay }}}
$$

\section{Data transmission and intra cluster adjustment}

When the cluster head finds that the backward ant arrives, the cluster head can send out the fused and cached information through the established routing information. The data forwarding formula between clusters is as follows:

$$
P_{i j}(t)=\frac{\left[\eta_{i j}(t)\right]^{(\beta)}}{\sum_{n \in k}\left[\eta_{i n}(t)\right]^{(\beta)}}
$$

The algorithm uses the "round" mechanism, each round needs to be re clustered to establish the route, which will consume a lot of energy, so it uses the method of adjusting within the cluster to re select the cluster head. At the end of a run, if the current energy of the cluster head is not less than the average energy of the cluster members, the cluster head does not need to change. On the contrary, adjust the cluster head and replace it with a node larger than the average energy of the cluster members. Then calculate and determine the final new cluster head according to equation (5). The new cluster head sends its own information to other nodes and informs them of the success of the election, and the ordinary nodes adjust the corresponding routing information after receiving it.

\section{EXPERIMENT AND ANALYSIS}

LEACH [7] is a typical clustering routing, and EEUC [2] is a classic representative of non-uniform clustering. The simulation and comparative experiments of LEACH, EEUC and UCMRA routing algorithms are carried out by using MATLAB platform. The experimental contents are the statistics of cluster head number distribution, node failure and average energy consumption. Some parameters in the experiment are shown in Table 2, and the experimental results are shown in Figures 1,2 and 3.

Table 2 simulation experiment parameters

\begin{tabular}{|c|c|c|c|}
\hline Parameter name & value & Parameter name & value \\
\hline Area size & $200 \mathrm{~m} \times 200 \mathrm{~m}$ & $d_{0}$ & $87 \mathrm{~m}$ \\
Number of nodes & 400 & $q_{1}$ & 0.5 \\
Packet size & $4000 \mathrm{bit}$ & $q_{2}$ & 0.5 \\
Base station location & $(100,250)$ & $c_{1}$ & 0.5 \\
Node initial energy & $0.5 \mathrm{~J} / \mathrm{node}$ & $c_{2}$ & 0.5 \\
$E_{\text {elec }}$ & $50 \mathrm{~nJ} / \mathrm{bit}$ & $R_{\max }$ & $90 \mathrm{~m}$ \\
$\varepsilon_{f s}$ & $10 \mathrm{pJ} / \mathrm{bit} / \mathrm{m}^{2}$ & $\alpha$ & 0.5 \\
$\varepsilon_{c m p}$ & $0.0013 \mathrm{pJ} / \mathrm{bit}^{\mathrm{m}} / \mathrm{m}^{4}$ & $\beta$ & 0.5 \\
\hline
\end{tabular}


As shown in Figure 1, the distribution of LEACH cluster heads in figure $\mathrm{A}$ is relatively unstable and fluctuates greatly, while EEUC in Figure B and UCMRA in Figure $\mathrm{C}$ are relatively stable, and the latter tends to be normal distribution. Clustering method plays an important role in the result of cluster head distribution. LEACH uses the method of random number and threshold to compare, so it is difficult to control the stability of cluster head distribution. EEUC algorithm uses the way of election cluster head, first select the candidate cluster head and then determine the final cluster head, in order to avoid the randomness of selection, the selected cluster head is better. Compared with EEUC, UCMRA algorithm not only includes the node energy factor, but also adds the node density factor, which makes the distribution of cluster heads more stable. After the cluster heads are selected successfully, it does not need to inform the whole network, only needs to inform the ordinary members within the competition radius, so as to reduce part of the energy consumption.

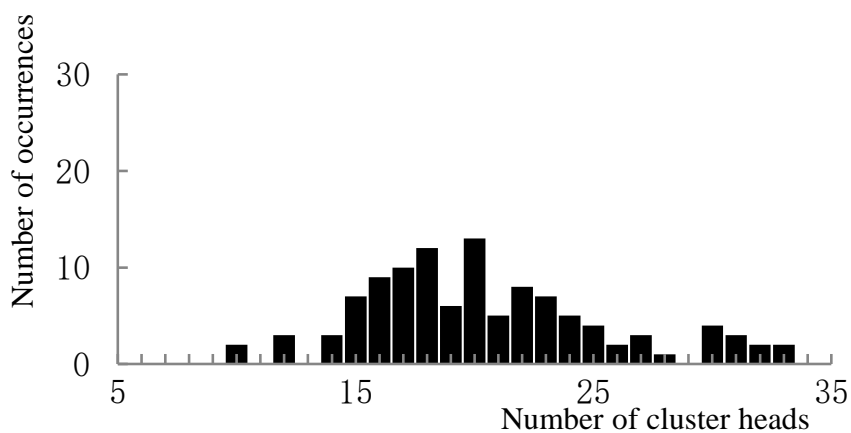

( A ) LEACH

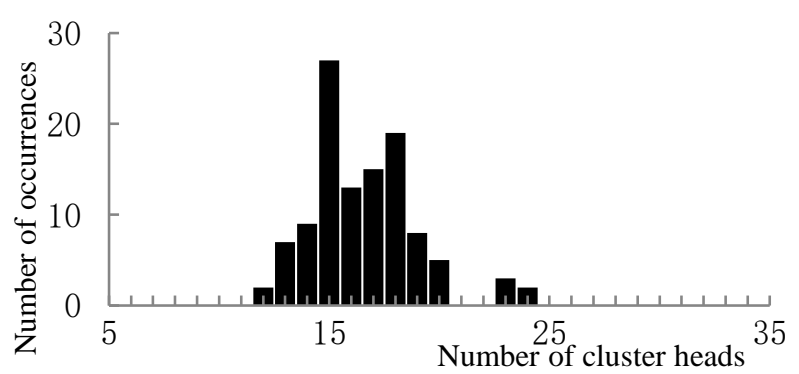

(B)EEUC

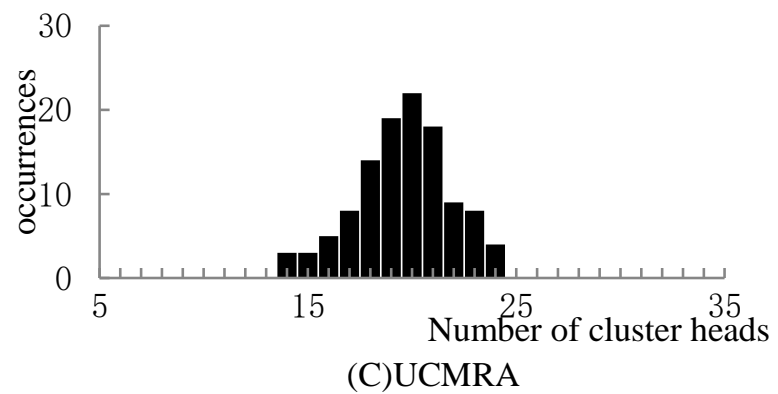

Figure 1 Statistics of cluster head number distribution.

As can be seen from Figure 2, LEACH algorithm generates failure nodes from 400 rounds of network operation, EEUC generates failure nodes from around 700 rounds, while UCMRA algorithm generates failure nodes from 1000 rounds. Nearly half of leach nodes fail in 500 rounds, while the survival rate of the other two routes is still $100 \%$. About 1200 rounds of EEUC nodes have all died, while about 1400 rounds of UCMRA nodes have all died. It can be seen that the network life of UCMRA is nearly $17 \%$ longer than that of EEUC.

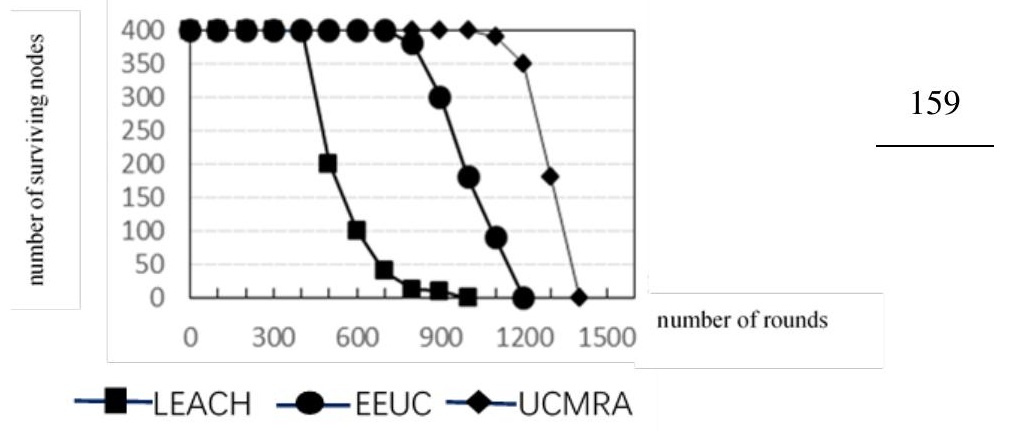

Figure 2 Comparison of node survival number

As can be seen from Figure 3, the initial energy value of the network is 200J, and the energy value of LEACH algorithm drops to 0 in about 1000 rounds. Compared with LEACH and EEUC, UCMRA has a lower energy consumption decline rate. This is because UCMRA not only optimizes the selection method of cluster heads and selects better cluster heads, but also optimizes the energy path between cluster heads in combination with ant colony algorithm, So that the limited energy in the network can be used efficiently. 


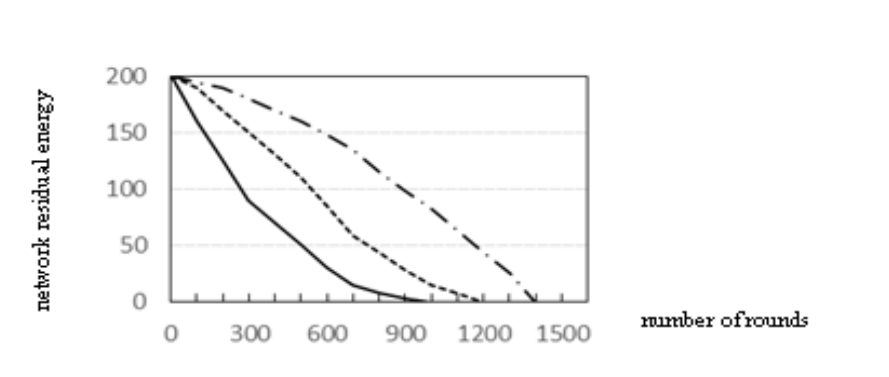

LEACH

$$
\text { EEUC - . UCMRA }
$$

Figure 3 Comparison of network residual energy

\section{CONCLUSION}

This paper proposes UCMRA algorithm, which combines the non-uniform clustering method and ant colony algorithm to achieve efficient and energy-saving multipath routing. In this algorithm, candidate nodes are selected by probability and cluster heads are selected by selection method. The optimized ant colony algorithm is used to construct multi hop routing. Simulation results show that UCMRA not only saves energy, but also significantly alleviates the "hot zone" problem and prolongs the effective working time of the whole network.

\section{REFERENCES}

[1] DO R IGOM, GAMBA R DELLALM. Ant colony system: A cooperative learning approach to the traveling salesman problem [ J] .IEEE Trans Evolution Computation, 1997, 1(1):53-66.

[2] Li Chengfa, Chen guihai, Ye Mao, et al. A wireless sensor network routing association based on heterogeneous clustering [J]. Journal of computer, 2007, 30 (01): 27-36.

[3] Miao congcongcong, Cen qingkui, Cao jianwei, Zhang gang. Energy balance and nonuniform clustering routing algorithm of wireless sensor network based on ant colony [J]. Computer application, 2013, 33 (12): 3410-3414.

[4] Zhang Wenmei, Liao fubao. Improved routing algorithm for nonuniform clustering in wireless sensor networks [J]. Journal of sensing technology, 2015, 28 (05): 739-743.

[5] Niutao, Zhuangyi, Fengning, Yu Yao Bing, Gu Jingjing. Nonuniform clustering routing algorithm for wireless sensor networks based on ant colony optimization [J]. Small microcomputer system, 2016, (01): 72-77.

[6] Liu Guofan, many. WSN routing protocol based on heterogeneous clustering and path optimization [J]. Computer engineering and science, 2015, 37 (08): 1492-1497.

[7] Heinzelman W,Chandrakasan A,Balakrishnan H.Energy-efficient communication protocol for wireless micro-sensor networks[C]// Proc of the 33rd Hawaii International Conference on System Sciences.Washington,IEEE Computer Society,2000:3005-3014. 